\title{
Experimental Realization of an Exact Solution to the Vlasov Equations for an Expanding Plasma
}

\author{
S. Laha, ${ }^{1}$ P. Gupta, ${ }^{1}$ C. E. Simien,,${ }^{1}$ H. Gao, ${ }^{1}$ J. Castro, ${ }^{1}$ T. Pohl, ${ }^{2}$ and T. C. Killian ${ }^{1}$ \\ ${ }^{1}$ Department of Physics and Astronomy, Rice University, Houston, Texas 77005, USA \\ ${ }^{2}$ ITAMP, Harvard-Smithsonian Center for Astrophysics, 60 Garden Street, Cambridge, Massachusetts 02138, USA
}

(Received 14 May 2007; published 8 October 2007)

\begin{abstract}
We study the expansion of ultracold neutral plasmas in the regime in which inelastic collisions are negligible. The plasma expands due to the thermal pressure of the electrons, and for an initial spherically symmetric Gaussian density profile, the expansion is self-similar. Measurements of the plasma size and ion kinetic energy using fluorescence imaging and spectroscopy show that the expansion follows an analytic solution of the Vlasov equations for an adiabatically expanding plasma.
\end{abstract}

DOI: 10.1103/PhysRevLett.99.155001

PACS numbers: 52.38.Kd, 52.27.Gr, 52.65.Ff

Exactly solvable problems are rare in physics and serve as ideal models that provide intuition for understanding more complex systems. Here, we report the experimental realization of a laser-produced plasma whose dynamics can be described by an analytic solution to the Vlasov equations [1,2], which are central equations in the kinetic theory of plasmas. Expansion into a surrounding vacuum is fundamentally important and typically dominates the dynamics of laser-produced plasmas [3], such as in inertial confinement fusion experiments [4], x-ray lasers [5], or production of energetic $(>\mathrm{MeV})$ ions through irradiation of solids [6,7], foils [8-14], and clusters [15].

We study plasma expansion with ultracold neutral plasmas (UNPs) [16], which are created by photoionizing laser-cooled strontium atoms [17] just above the ionization threshold. The well-controlled initial conditions and relatively slow dynamics of these systems [17,18] provide distinct advantages for studying this problem. In addition, we use fluorescence imaging and spectroscopy for the first time in UNPs. Although the typical energy and density of UNPs is much lower than in traditional hot plasmas produced by intense laser irradiation, the ratio of electron kinetic to potential energy and the resulting expansion dynamics is similar.

The investigation of plasma expansion dates back many decades $[19,20]$. Recently, exact solutions for spatially finite plasmas expanding into vacuum were identified for one-dimensional plasmas [1] and later extended to three dimensions $[2,21]$. This work was motivated by plasmas produced with short-pulse lasers.

The Vlasov equations, along with Poisson's equation, describe the evolution of electron $(\alpha=e)$ and ion $(\alpha=i)$ distribution functions, $f_{\alpha}(\mathbf{r}, \mathbf{v})$. The Vlasov equations neglect radiative processes and collisional phenomena such as electron-ion thermalization and three-body recombination [22], but they describe many types of plasmas and are part of the foundation of kinetic theory.

Among broad classes of general analytic solutions to the Vlasov equations [21], UNPs realize a particular solution that is valid for a quasineutral plasma with spherically symmetric Gaussian distribution functions

$$
f_{\alpha} \propto \exp \left[-\frac{r^{2}}{2 \sigma^{2}}-\frac{m_{\alpha}(\mathbf{v}-\mathbf{u})^{2}}{2 k_{B} T_{\alpha}}\right] .
$$

Quasineutrality is defined by $n_{e} \approx n_{i}$, where electron and ion densities are $n_{\alpha}(\mathbf{r})=\int d \mathbf{v} f_{\alpha}(\mathbf{r}, \mathbf{v}) . T_{\alpha}$ are electron and ion temperatures, and the local average velocity varies in space according to $\mathbf{u}(\mathbf{r}, t)=\gamma(t) \mathbf{r}$. The temperatures must scale as $\sigma^{2} T_{\alpha}=$ const [1], which is expected for adiabatic cooling in a spherical UNP $[23,24]$.

Under these conditions, the plasma dynamics is given by $T_{\alpha}(t)=T_{\alpha}(0) /\left(1+t^{2} / \tau_{\exp }^{2}\right)$, where the characteristic expansion time $\tau_{\exp }$ is given by $\tau_{\exp }=$ $\sqrt{m_{i} \sigma(0)^{2} / k_{B}\left[T_{e}(0)+T_{i}(0)\right]}$. Also,

$$
\sigma(t)^{2}=\sigma(0)^{2}\left(1+t^{2} / \tau_{\exp }^{2}\right)
$$

and

$$
v_{i, \mathrm{rms}}=\sqrt{\frac{k_{B}}{m_{i}}\left\{\frac{t^{2}}{\tau_{\exp }^{2}}\left[T_{e}(t)+T_{i}(t)\right]+T_{i}(t)\right\}}
$$

describe the evolution of the characteristic plasma size and ion velocity. We define the rms one-dimensional ion velocity $\sqrt{\left\langle(\mathbf{v} \cdot \hat{\mathbf{y}})^{2}\right\rangle} \equiv v_{i, \text { rms }}$, where $\hat{\mathbf{y}}$ is the laser propagation direction, $\mathbf{v}$ is the total ion velocity including random thermal motion and expansion, and angled brackets refer to an average over the distribution function.

The expansion is self-similar because thermal pressure produces a radial acceleration given by $[2,24]$

$$
\dot{\mathbf{u}}=-\frac{k_{B}\left[T_{e}(t)+T_{i}(t)\right]}{m_{i}} \frac{\nabla n(r, t)}{n(r, t)}=\frac{k_{B}\left[T_{e}(t)+T_{i}(t)\right]}{m_{i} \sigma(t)^{2}} \mathbf{r} .
$$

The simplification implied by the last equality is only valid for a spherical Gaussian plasma, and the linearity in $\mathbf{r}$ preserves the shape of the distribution functions.

Plasmas produced with solid targets, foils, and clusters are often quasineutral and well-described by the Vlasov 
equations, and electrons typically cool adiabatically after the laser is extinguished [12,13], but experimental conditions studied are typically very complicated and evolve extremely rapidly, which frustrates detailed comparison between experiment and theory. Final ion kinetic energy distributions have been shown to agree with simple models $[12,15]$, but in general, these systems lack the Gaussian distribution functions necessary to realize the analytically describable self-similar expansion [13].

For appropriate initial conditions, UNPs fulfill the requirements for the analytic solution. In general, UNP peak densities are on the order of $10^{15} \mathrm{~m}^{-3}$, and the profile follows that of the laser-cooled atom cloud, which we adjust to be a spherically symmetric Gaussian. The ion temperature is about $1 \mathrm{~K}$ determined by disorder-induced heating $[17,25]$. Electron temperatures can range from 1 to $1000 \mathrm{~K}$ and are set by the detuning, $E_{e}$, of the ionization laser above threshold. For this study, we use intermediate initial electron temperatures $(\sim 100 \mathrm{~K})$, which at this density are high enough to avoid inelastic collisional and radiative processes [23,26-28] but low enough to maintain quasineutrality [29]. The photoionization pulse length $(\sim 10 \mathrm{~ns})$ is much less than the expansion time scale $(\sim 10 \mu \mathrm{s})$.

The electron distribution equilibrates locally within $100 \mathrm{~ns}$ and globally within $1 \mu \mathrm{s}$ after photoionization $[23,24]$. This ensures a Gaussian electron distribution function at the start of the expansion. Despite these very rapid electron-electron collisions the corresponding collision integral vanishes for the spherically symmetric, Gaussian velocity distribution Eq. (1). Hence the highly collisional UNP considered here provides an ideal model system for truly collisionless plasmas behavior.

Ions reach local thermal equilibrium within a few $100 \mathrm{~ns}$ [17]. They do not equilibrate globally on the time scale of the expansion [18], but the ions are so cold compared to the electrons that the lack of a global ion temperature does not cause any significant deviation from the exact solution. The low ion temperature also implies that the ions form a strongly coupled fluid $[17,30]$, which, however, negligibly affects the plasma expansion [24].

The analytic expansion solution has been discussed previously for UNPs [23,24], and it has been checked against average terminal ion expansion velocities [26] and measured electron temperatures [31] that qualitatively affirm the importance of adiabatic cooling for appropriate initial conditions. The lack of spatial and temporal resolution, however, has prevented conclusive tests of the analytic predictions. Cummings et al. [32] adapted the formalism of Refs. [23,24] and used light scattering from a small region of the plasma to study the expansion of ultracold plasmas with an elongated aspect ratio, but they found significant deviations from the predictions of the model that perhaps arose because the condition of spherical symmetry was not fulfilled.

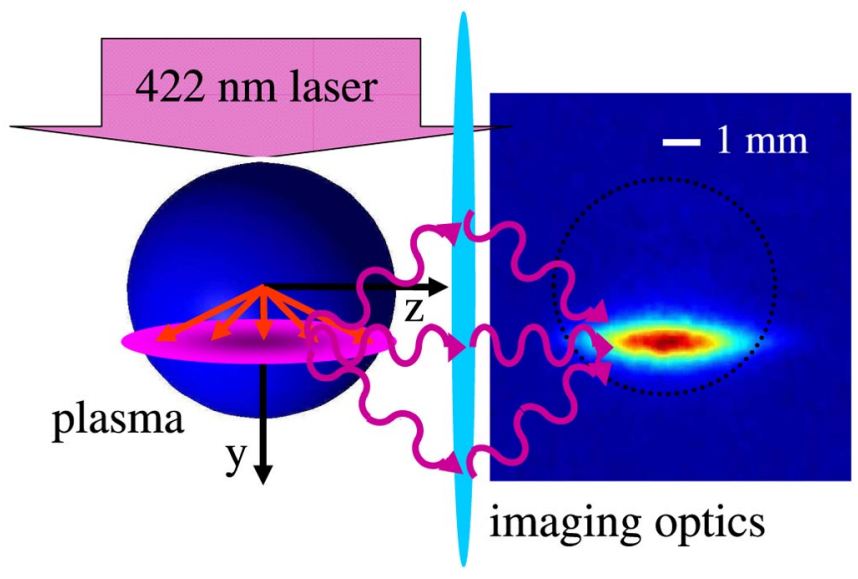

FIG. 1 (color online). Recording fluorescence of UNPs. Correlation between position and expansion velocity [arrows (red online)] produces a striped image when Doppler-shift due to expansion exceeds Doppler broadening from thermal ion velocity. The number of ions in this plasma is $2 \times 10^{8}$, and it can be calibrated absolutely with absorption imaging [17]. The fluorescence laser is blue-detuned by $193 \mathrm{MHz}$ from resonance. We collect $1 \%$ of the scattered photons due to solid angle limitations. The theoretically expected resolution is $40 \mu \mathrm{m}$ for our optical setup.

Figure 1 shows a schematic of the fluorescence imaging experiment. A laser beam that is near resonance with the ${ }^{2} S_{1 / 2}-{ }^{2} P_{1 / 2}$ transition in $\mathrm{Sr}^{+}$at $\lambda=422 \mathrm{~nm}$ propagates along $\hat{y}$ and illuminates the plasma. Fluorescence in a perpendicular direction $(\hat{z})$ is imaged with a 1:1 relay telescope onto an image-intensified CCD camera. The $422 \mathrm{~nm}$ light is typically applied in a $1 \mu$ s pulse to provide temporal resolution, with an intensity of a few $\mathrm{mW} / \mathrm{cm}^{2}$.

A general expression for the fluorescence is

$$
\begin{aligned}
F(\nu, x, y) \propto & \int d s \frac{1}{1+\left[\frac{2(\nu-s)}{\gamma_{\text {eff }} / 2 \pi}\right]^{2}} \times \int \frac{d z n(\mathbf{r})}{\sqrt{2 \pi} \sigma_{D}\left[T_{i, \text { therm }}(\mathbf{r})\right]} \\
& \times \exp \left\{-\frac{\left[s-\left(\nu_{0}+\nu_{\exp }^{y}(\mathbf{r})\right)\right]^{2}}{2 \sigma_{D}^{2}\left[T_{i, \text { therm }}(\mathbf{r})\right]}\right\}
\end{aligned}
$$

where $\nu$ is the laser frequency and $\gamma_{\text {eff }}=\gamma_{0}+\gamma_{\text {laser }}$ is the sum of the natural linewidth of the transition $(2 \pi \times$ $20 \mathrm{MHz})$ and the imaging laser linewidth $(2 \pi \times 8 \mathrm{MHz})$. $T_{i \text {,therm }}(\mathbf{r})$ is the local temperature of the ions describing random thermal motion, which gives rise to the Doppler width $\sigma_{D}$. Because of the directed expansion velocity, the average resonance frequency of the transition for atoms at $\mathbf{r}$ is Doppler-shifted from the unperturbed value, $\nu_{0}$, by $\nu_{\exp }^{y}(\mathbf{r})=\mathbf{u}(\mathbf{r}) \cdot \hat{y} / \lambda$. The spatial variation in $T_{i, \text { therm }} \sim$ $n_{i}^{1 / 3}[18]$ is small compared to the directed expansion energy, so $T_{i, \text { therm }}$ can be taken as constant.

Summing a series of images taken at equally spaced frequencies covering the entire ion resonance is equivalent to integrating $F(\nu, x, y)$ over frequency. This yields a signal proportional to the areal plasma density, $\int n(x, y, z) d z$, 
which for a Gaussian density distribution should take the form $n_{\text {areal }}(x, y)=\sqrt{2 \pi} \sigma n_{0} \exp \left[-\left(x^{2}+y^{2}\right) / 2 \sigma^{2}\right]$.

Figure 2 shows that Gaussian fits of the areal density are excellent during more than a factor of 2 change in $\sigma$ from the earliest times until the signal expands beyond the range of the imaging system. This provides direct confirmation of the self-similar nature of the expansion.

There is no sign of deviation from Gaussian shape at large radius. This might seem surprising because selfsimilarity follows from Eq. (4), and this equation must break down at large $r$ where it implies unphysical accelerations arising from unphysically large electric fields. One expects breakdown where the plasma is not quasineutral, which occurs when the local Debye screening length exceeds the length scale for ion density variation, or $\sigma<$ $\lambda_{D}(r)=\left[\varepsilon_{0} k_{B} T_{e} / n_{e}(r) e^{2}\right]^{1 / 2}$ for a Gaussian plasma. At the radius where $\sigma \approx \lambda_{D}(r)$, which is typically $r \sim 4 \sigma$ for UNPs, the accelerating electric field reaches a maximum of $\sim k_{B} T_{e} / e \lambda_{D}$, identical to peak accelerating fields in plasmas generated from foils [14]. Peaks in the electric field can lead to wave breaking, shock waves, and the formation of an ion front as seen in theories for UNPs $[23,24]$ and traditional short-pulse laser-produced plasmas $[13,33]$, and can determine the maximum ion kinetic energy [12]. Lack of such features in data such as Fig. 2 confirms the extent of quasineutrality one would expect for such a low electron temperature [23] and may also suggest that finite ion temperature and strong coupling effects damp wave breaking at the plasma edge [24].

The evolution of $\sigma$ (Fig. 3) can be extracted from fits such as Fig. 2. Again, data evolve as predicted by theory. Higher electron temperature and smaller size lead to a faster expansion because this increases the thermal pres-
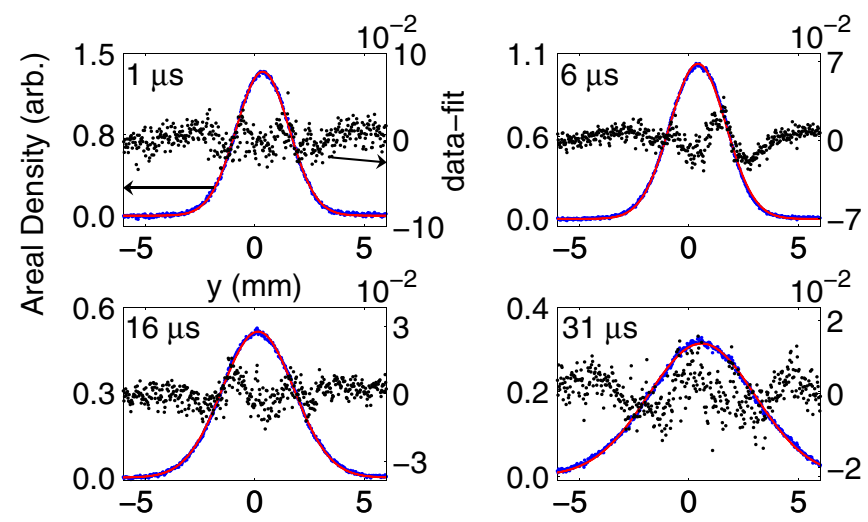

FIG. 2 (color online). Evolution of the areal density for a plasma with $1.6 \pm 0.1 \times 10^{8}$ ions, $2 E_{e} / 3 k_{B}=48 \pm 3 \mathrm{~K}$, and $\sigma(0)=1.3 \pm 0.1 \mathrm{~mm}$. The areal density (arbitrary units) is found by summing together 50 images taken at equally spaced frequencies that fully cover the ion resonance. The Gaussian fits (solid line) to linear cuts show that the expansion is self-similar. The time indicated is the evolution time since plasma creation. The right-hand axes show that the differences between data and fit are small. sure [Eq. (4)]. Fits of the data using Eq. (2) yield values of $T_{e}(0)$ that agree reasonably well with the expected values of $2 E_{e} / 3 k_{B}$. This confirms that on the time scale of the expansion there are no significant collisional or radiative processes changing the electron temperature such as threebody recombination or electron-ion thermalization, which is assumed in the Vlasov equations.

To completely characterize the plasma expansion we also measure the light-scattering resonance spectrum, formed from the integrated fluorescence in each of a series of images taken at different frequencies [Fig. 4(a)]. Because of expansion velocity, $\mathbf{u}(\mathbf{r})$, the resulting signal $\left[\int d x d y F(\nu, x, y)\right]$ is a Voigt profile. The rms width of the Gaussian component of this profile arising from Doppler broadening reflects both thermal ion motion and directed expansion and is given by $\tilde{\sigma}_{D}=v_{i, \mathrm{rms}} / \lambda$.

Equation (3) provides an excellent fit to the data, and the extracted values of $T_{e}(0)$ are consistent with $2 E_{e} / 3 k_{B}$ within experimental uncertainty. The small initial offset of $v_{i, \text { rms }}$ is due to disorder-induced heating of the ions within the first microsecond [17], which locally produces a thermal ion velocity distribution. $v_{i, \text { rms }}$ quickly increases well above this value as electron pressure drives the plasma expansion and electron thermal energy is converted into radial ion velocity. As $\sigma$ increases and the electrons cool adiabatically, the acceleration decreases [Eq. (4)]. The ions reach terminal velocity when essentially all electron kinetic energy is transferred to the ions.

Measured density profiles (Fig. 2) confirm the validity of Eq. (4), which shows that ion acceleration is sensitive to the instantaneous electron temperature and width of the plasma. Agreement between experiment and theory for

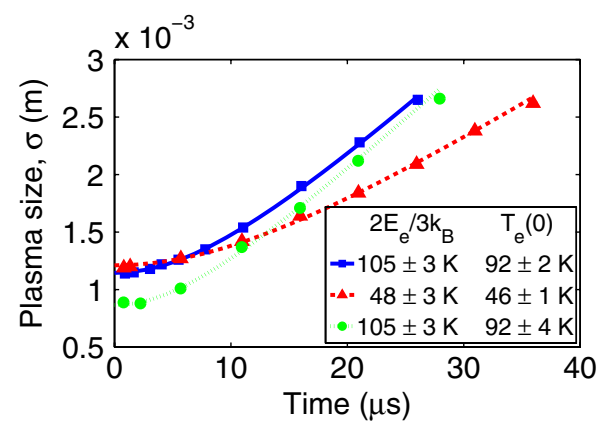

FIG. 3 (color online). Evolution of the plasma size, extracted from fits such as in Fig. 2. $T_{e}(0)$ and $\sigma(0)$ are fit, while $T_{i}(0)$ is taken from the theoretical expression for disorder-induced heating $[17,25]$. Uncertainties in $2 E_{e} / 3 k_{B}$ reflect 1 standarddeviation calibration uncertainty in the wavelength of the photoionizing laser. Quoted uncertainties in $T_{e}(0)$ are statistical, but there is an additional systematic uncertainty of a few percent arising from calibration of the imaging-system magnification and overlap of the plasma and fluorescence excitation laser. Statistical uncertainty in the measurement of $\sigma$ is less than the size of the plotting symbols. Initial peak densities for these samples are $\sim 10^{16} \mathrm{~m}^{-3}$. 

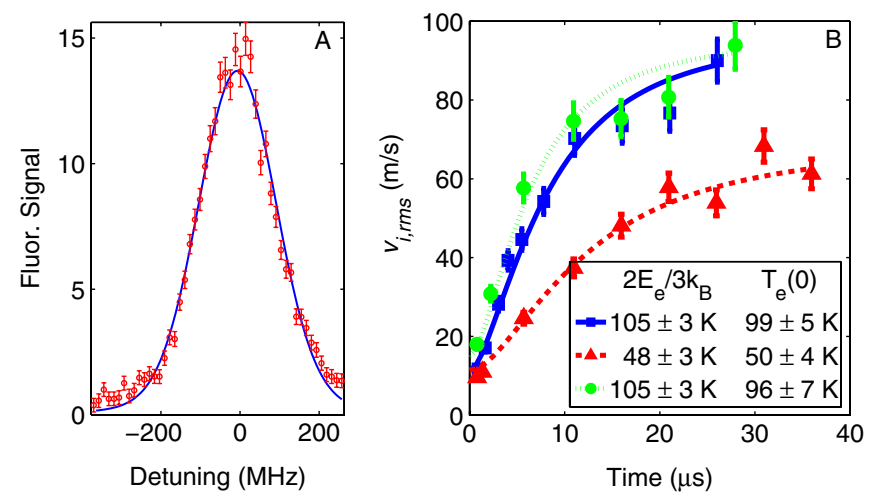

FIG. 4 (color online). (a) Sample spectrum and (b) evolution of the rms ion velocity $\left(v_{i, \mathrm{rms}}\right)$. (a) Spectrum for $2 E_{e} / 3 k_{B}=$ $105 \pm 3 \mathrm{~K}$, peak ion density of $3 \times 10^{15} \mathrm{~m}^{-3}$, and plasma evolution time of $3 \mu \mathrm{s}$. Fits to a Voigt profile provide $v_{i, \mathrm{rms}}$. (b) The velocity evolution is fit well by Eq. (3) with $T_{e}(0)$ as a fit parameter, $\sigma(0)$ determined from the cloud size measurements (Fig. 3), and $T_{i}(0)$ taken from the theoretical expression for disorder-induced heating $[17,25]$.

both the size evolution and the ion velocity dynamics hence demonstrates that $T_{e}(t)$ also follows the dynamics predicted by the analytic solution.

We have demonstrated a plasma in which the expansion matches an analytic solution to the Vlasov equations [2] proposed as a basic model for quasineutral laser-produced plasmas. To realize this situation, it is necessary to create UNPs with a spherical Gaussian density distribution and appropriate density and electron temperature to avoid inelastic collisions that would heat electrons or lead to electron-ion equilibration. The expansion dynamics shown here provide a general tool for developing understanding and intuition for complex systems and provide insight into the implications when initial conditions render the analytic solution invalid. Important examples are higher energy plasmas that evolve rapidly and have initial geometries that are difficult to model, or UNPs with lower initial $T_{e}$ and higher density in which inelastic collisions can increase electron temperature by an order of magnitude or more [29].

This work was supported by the National Science Foundation (Grants No. PHY-0355069 and No. PHY0140320) and the David and Lucille Packard Foundation.
[1] A. V. Baitin and K. M. Kuzanyan, J. Plasma Phys. 59, 83 (1998).

[2] D. S. Dorozhkina and V.E. Semenov, Phys. Rev. Lett. 81, 2691 (1998).

[3] M. D. Perry and G. Mourou, Science 264, 917 (1994).

[4] J. Lindl, Phys. Plasmas 2, 3933 (1995).

[5] H. Daido, Rep. Prog. Phys. 65, 1513 (2002).

[6] E. L. Clark et al., Phys. Rev. Lett. 85, 1654 (2000).

[7] R. A. Snavely et al., Phys. Rev. Lett. 85, 2945 (2000).

[8] A. Maksimchuk et al., Phys. Rev. Lett. 84, 4108 (2000).

[9] J. Badziak et al., Phys. Rev. Lett. 87, 215001 (2001).

[10] J. Fuchs et al., Phys. Rev. Lett. 94, 045004 (2005).

[11] L. Romagnani et al., Phys. Rev. Lett. 95, 195001 (2005).

[12] M. Murakami et al., Phys. Plasmas 12, 062706 (2005).

[13] P. Mora, Phys. Plasmas 12, 112102 (2005).

[14] T. Grismayer and P. Mora, Phys. Plasmas 13, 032103 (2006).

[15] D. R. Symes, M. Hohenberger, A. Henig, and T. Ditmire, Phys. Rev. Lett. 98, 123401 (2007).

[16] T. C. Killian, Science 316, 705 (2007).

[17] C. E. Simien et al., Phys. Rev. Lett. 92, 143001 (2004).

[18] Y. C. Chen et al., Phys. Rev. Lett. 93, 265003 (2004).

[19] A. V. Gurevich, L. V. Pariskaya, and L. P. Pitaievskii, Sov. Phys. JETP 22, 449 (1966).

[20] C. Sack and H. Schamel, Phys. Rep. 156, 311 (1987).

[21] V.F. Kovalev and V. Y. Bychenkov, Phys. Rev. Lett. 90, 185004 (2003).

[22] P. Mansbach and J. Keck, Phys. Rev. 181, 275 (1969).

[23] F. Robicheaux and J. D. Hanson, Phys. Plasmas 10, 2217 (2003).

[24] T. Pohl, T. Pattard, and J. M. Rost, Phys. Rev. A 70, 033416 (2004).

[25] M. S. Murillo, Phys. Rev. Lett. 87, 115003 (2001).

[26] S. Kulin, T. C. Killian, S. D. Bergeson, and S. L. Rolston, Phys. Rev. Lett. 85, 318 (2000).

[27] S. G. Kuzmin and T. M. O’Neil, Phys. Plasmas 9, 3743 (2002).

[28] S. Mazevet, L. A. Collins, and J. D. Kress, Phys. Rev. Lett. 88, 055001 (2002).

[29] P. Gupta et al., Phys. Rev. Lett. 99, 075005 (2007).

[30] S. Ichimuru, Rev. Mod. Phys. 54, 1017 (1982).

[31] J. L. Roberts, C. D. Fertig, M. J. Lim, and S. L. Rolston, Phys. Rev. Lett. 92, 253003 (2004).

[32] E. A. Cummings, J.E. Daily, D.S. Durfee, and S.D. Bergeson, Phys. Plasmas 12, 123501 (2005).

[33] A. E. Kaplan, B. Y. Dubetsky, and P. Shkolnikow, Phys. Rev. Lett. 91, 143401 (2003). 